\title{
Perspectivas de las investigaciones en torno al conocimiento indígena y las TIC: Un enfoque de-colonial
}

\author{
Research Perspectives on Indigenous Knowledge and ICT: A Decolonial Approach
}

\section{Perspectivas de investigação sobre o conhecimento indígena e as TICs: Uma abordagem decolonial}

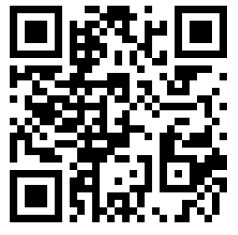

\author{
Gloria Flores-Fuentes \\ Bachillerato General Oficial Benito Juárez \\ Puebla, México \\ gloriafloresfuentes17@gmail.com \\ http://orcid.org/0000-0002-8762-0469 \\ Yadira Navarro-Rangel \\ Benemérita Universidad Autónoma de Puebla \\ Puebla, México \\ ynavarro44@gmail.com \\ http://orcid.org/0000-0001-8553-6303
}

Recibido $\cdot$ Received $\cdot$ Recebido: 08 / 06 / 2018
Corregido $\cdot$ Revised $\cdot$ Revisado: 13 / 10 / 2019
Aceptado $\cdot$ Accepted $\cdot$ Aprovado: 14/ 02 / 2020

Resumen: El objetivo de la presente comunicación es mostrar los resultados de una revisión bibliográfica en torno a las investigaciones y experiencias sobre el conocimiento indígena y las TIC para el periodo 2006-2017. La búsqueda se limitó a los casos en que las TIC fueron usadas como un medio de preservar, transmitir o gestionar el conocimiento indígena. Si bien es cierto que existen diversas ópticas hacia el objeto de estudio tales como las TIC usadas como medio de comunicación de los grupos indígenas, por ejemplo, el caso del movimiento indígena en internet (Monasterios, 2001), o el uso de internet para promover sus productos (http://www.tosepan.com/; http:// chiapasproject.com/sp/proyecto-de-escuela/las-mujeres/mujeres-de-maiz/L, por citar algunos, no es intención del presente análisis estudiar dichas perspectivas. Los resultados del análisis muestran cinco tendencias en la investigación: Creación de contenido digital cultural e inclusión digital; preservación del conocimiento indígena; gestión del conocimiento indígena; conocimiento indígena y TIC en procesos educativos, y diseño de prototipos tecnológicos (Software, plataformas virtuales y aplicaciones móviles) acordes al contexto indígena. En esta última línea de investigación, se identificó que los estudios están orientados hacia la adecuación del diseño de prototipos al contexto y que se fundamentan en un tercer paradigma emergente derivado de las investigaciones referidas a la interacción ser humano-computador (IHC): la matriz fenomenológica, también conocida como Digital Ground. Se concluye que la dimensión diseño de prototipos tecnológicos acordes al contexto indígena es la más relevante en tanto que aglutina a las otras cuatro dimensiones y, al mismo tiempo, se vislumbra una posible área de desarrollo a partir de la conjunción de los temas de la gestión, 
http://doi.org/10.15359/ree.24-2.6

ROR: https://ror.org/01t466c14 Universidad Nacional, Costa Rica

http://www.una.ac.cr/educare

educare@una.cr

educación y TIC, centrada en la preservación del conocimiento indígena, lo que permitiría empoderar a las comunidades indígenas. Adicionalmente, se encontró que las investigaciones se hallan en mayor medida en África, son aún incipientes en Latinoamérica, por lo que se detecta la necesidad de abonar en este tipo de estudios que contribuyan en el saber y comprensión de los sistemas de conocimientos indígenas y su fortalecimiento usando las TIC.

Palabras claves: Sistemas de conocimiento indígena; transferencia del conocimiento; TIC.

Abstract: This essay aims to show the results of a bibliographic review of research and experiences on indigenous knowledge and ICT for the 2006-2017 period. The study focused on cases in which ICT was used to preserve, transmit, or manage indigenous knowledge. Although it is true that there are different approaches to the object of study such as ICT used as a means of communication for indigenous groups, such is the case of the indigenous movement on the Internet (Monasterios, 2001), or the use of the Internet to promote their products (http://www.tosepan.com/; http://chiapasproject. $\mathrm{com} / \mathrm{sp} /$ proyecto-de-escuela/las-mujeres/mujeres-de-maiz/), to cite some examples, this paper does not intend to analyze these approaches. As results, the research revealed the following five trends: the creation of digital cultural content and digital inclusion; preservation of indigenous knowledge; management of indigenous knowledge; indigenous knowledge and ICT in educational processes and design of technological prototypes (software, virtual platforms, and mobile applications) in consonance with the indigenous context. In this last line of research, it was identified that the investigations are oriented towards the adequacy of the design of prototypes to the context, and that they are based on a third emergent paradigm derived from the inquiry referred to the HumanComputer Interaction $(\mathrm{HCl})$ : the phenomenological matrix, also known as Digital Ground. It is therefore concluded that the Technological Prototype Design dimension, in accordance with the indigenous context, is the most relevant one since it encompasses the other four dimensions. At the same time, a likely area of development is envisaged from the conjunction of management, education, and ICT; this area is focused on the preservation of indigenous knowledge that would empower indigenous communities. Besides, the results of the review indicate that research on indigenous knowledge and ICT is mostly developed in Africa; it is still incipient in Latin America. Therefore, there is a need to pay for this type of study to contribute to the research and understanding of the indigenous knowledge systems and their strengthening by using ICT.

Keywords: Indigenous knowledge systems; knowledge transfer; ICT.

Resumen: O objetivo da presente comunicação é mostrar os resultados de uma revisão bibliográfica sobre as pesquisas e experiências relacionadas aos conhecimentos indígenas e TIC para o período 2006-2017. A busca foi limitada aos casos em que as TICs foram usadas como um meio de preservar, transmitir ou gerenciar o conhecimento indígena. Embora seja verdade que existem várias perspectivas em relação ao objeto de estudo, como as TIC usadas como meio de comunicação de grupos indígenas, por exemplo, o caso do movimento indígena na Internet (Monasterios, 2001) ou o uso da Internet para promover seus produtos (http://www.tosepan.com/; http://chiapasproject. com/sp/proyecto-de-escuela/las-mujeres/mujeres-de-maiz/, para citar alguns, esta não é a intenção deste trabalho. Os resultados da análise mostram cinco tendências em pesquisa: criação de conteúdo digital cultural e inclusão digital; preservação do conhecimento indígena; gestão do conhecimento indígena; conhecimento indígena e TIC nos processos educacionais e desenho 


\begin{abstract}
de protótipos tecnológicos (Software, plataformas virtuais e aplicativos móveis) de acordo com o contexto indígena. Nesta última linha de pesquisa, identificou-se que os estudos estão orientados para a adequação do desenho de protótipos para o contexto e que se baseiam em um terceiro paradigma emergente derivado de pesquisas relacionadas à interação humano-computador (IHC): a matriz fenomenológica, também conhecida como Digital Ground. Conclui-se que a dimensão de design dos protótipos tecnológicos de acordo com o contexto indígena é a mais relevante, pois reúne as outras quatro dimensões e, ao mesmo tempo, uma possível área de desenvolvimento é prevista a partir da conjunção de questões de gestão, educação e TIC, focadas na preservação do conhecimento indígena, o que permitiria capacitar comunidades indígenas. Além disso, verificouse que as pesquisas se concentram na África, e ainda são incipientes na América Latina, portanto, demonstra a relevância em investir nesse tipo de estudo capaz de contribuir para os sistemas de conhecimento indígenas e seu fortalecimento usando as TIC.
\end{abstract}

Palavras chave: Sistemas de conhecimento indígenas; transferência de conhecimento; TIC.

\title{
Introducción
}

El creciente desarrollo de las TIC ha permitido su inserción en diversos grupos sociales y culturales, ejemplo de ello son los grupos indígenas. La adopción de las TIC en los grupos indígenas ha suscitado el interés de las personas estudiosas respecto a la manera en que se apropian de ellas (Sandoval-Forero, 2013); los usos sociales que les dan (Gómez, 2005); o al diseño de prototipos acordes con su cosmovisión (Reitsma et al., 2013), por mencionar algunas líneas de investigación. El propósito de la revisión bibliográfica es identificar las investigaciones que aborden el tema del conocimiento indígena y su relación con las TIC. El análisis realizado en el presente trabajo pretende ubicar las investigaciones relacionadas en un tema poco explorado en México e incluso en Latinoamérica. El escrito está organizado de la siguiente forma: se presenta la metodología indicando las bases de datos, palabras clave, tipo de foro y las características de los cuadros a donde se concentró la información, posteriormente se hacen algunas aclaraciones conceptuales necesarias para contextualizarlo, después se plantea el análisis de la información, se muestran seis cuadros analíticos con las dimensiones encontradas y los principales aportes de cada dimensión, finalmente se exponen las conclusiones.

\section{Metodología}

La búsqueda se realizó en la Biblioteca Digital de la Benemérita Universidad Autónoma de Puebla (http://www.bibliotecas.buap.mx/), se consultó la base de datos de libre accesos Google Académico y las bases de datos Scopus, EBSCO, Science Direct y ACM. En Google Académico se encontraron trabajos alusivos a inclusión digital, creación de contenido digital cultural, apropiación tecnológica y TIC en procesos tecno-educativos; en Scopus, EBSCO, Science Direct y ACM se hallaron trabajos sobre gestión y preservación del conocimiento indígena y diseño de prototipos adecuados al contexto indígena. Las palabras clave usadas fueron "Indigenous Knowledge y ICT" y para Google Académico "conocimiento indígena y TIC". 
http://doi.org/10.15359/ree.24-2.6

ROR: https://ror.org/01t466c14 Universidad Nacional, Costa Rica

http://www.una.ac.cr/educare

educare@una.cr

Se identificaron 11 artículos, 12 conferencias, 1 simposio y 3 capítulos de libro. La base de datos que más abono en la búsqueda fue ACM (Association for Computing Machinery) y Science Direct y las revistas en las que se publicaron los trabajos son: International Journal of Human-Computer Studies, International Journal of Services Technology and Management, Pers Ubiquit Comput, Al y Soc, Procedia- Social and Behavioral Sciences, InterSedes, The Electronic Library, Guillermo de Ockham y Quinto Sol. Las conferencias provienen en su totalidad de la base de datos ACM y son: conferencia SAICSIT (Annual Conference of the South African Institute of Computer Scientists and Information Technologists) la que más publicó seguida de Conference on Human Factors in Computing Systems. Los capítulos de libro provienen de Human-Computer Interaction. Human-Centred Design Approaches, Methods, Tools, and Environments, Knowledge Management for Development y INTERACT 2011 (Conference on Human-Computer interaction), Part II, LNCS 6947.

Se elaboró un cuadro de doble entrada con la finalidad de organizar los trabajos de investigación, para ello se identificó el nombre de la revista, autoría, país de procedencia, año de publicación, sujeto de investigación, propósito del estudio, TIC utilizada, tipo de conocimiento indígena, metodología, referente teórico y finalmente los hallazgos.

\section{Aclaraciones conceptuales}

\section{Conocimiento indígena: Delimitaciones sobre el concepto y sus diversos enfoques}

El conocimiento indígena es conocido con diversos nombres, dependiendo del uso que se le da al tipo de conocimiento y en algunos casos de la ubicación geográfica, algunos nombres son: sabiduría popular, saber local, ciencia de lo concreto, conocimiento popular, local indigenous knowledge, conocimiento ecológico tradicional, por mencionar algunos (Pérez y Argueta, 2011).

En lo que concierne a este trabajo, la acepción que se usará es la de conocimiento indígena. Así mismo, el concepto de conocimiento indígena tiene diversas definiciones que estudios y organizaciones han proporcionado, por ejemplo, la Comisión Nacional para el Conocimiento y Uso de la Biodiversidad (CONABIO, 2008). No es objetivo del trabajo realizar un análisis exhaustivo de las definiciones, por lo que a continuación solo se presenta su definición:

Las poblaciones humanas en el transcurso de su historia han desarrollado conocimientos sofisticados sobre su entorno, sobre la flora y la fauna y sobre el uso de diversas especies. Esta experiencia adquirida durante miles de años, conocida como conocimiento ecológico tradicional, está estrechamente ligada al lenguaje, a las tradiciones orales y a la cosmovisión de cada población. La transmisión de este conocimiento se lleva a cabo mediante creencias, actitudes sociales, principios y convenciones de comportamiento y prácticas desarrolladas por la experiencia. (párr. 1) 
Adicional a las delimitaciones sobre el concepto conocimiento indígena y sus diversos enfoques, se encontró, en algunos artículos analizados, un paradigma emergente dominante que se aplica en las investigaciones relativas a la interacción sujeto humano-computador $(\mathrm{HCl}$ por sus siglas en inglés), por lo que a continuación se describe brevemente dicho paradigma.

\section{Paradigmas dominantes en las investigaciones interacción sujeto humano-computador $(\mathrm{HCl})$}

Harrison et al. (2007) identificaron 3 paradigmas dominantes en el contexto de las investigaciones interacción humano-computador. El primer paradigma es la unión entre factores de ingeniería y sujetos humanos; en este paradigma la interacción estudia la forma de acoplamiento ser humano-máquina, el objetivo de las investigaciones con el enfoque de este paradigma es la optimización de la interacción ser humano-computadora.

El segundo paradigma se organiza en torno a la metáfora central de que la mente y la computadora son como procesadores de información simétricos unidos. En este paradigma, las ciencias cognitivas abonan en tanto que sus avances y resultados pueden ser aplicados en el desarrollo de prototipos computacionales.

El tercer paradigma emergente, denominado matriz fenomenológica, está enfocado en embodied interaction'. Embodied interaction se fundamenta en la creación, manipulación y el intercambio de significados a través de la interacción acoplada por artefactos, es acerca de las relaciones entre la acción y su significado (Dourish, 2004). Este tercer paradigma emergente es el que se aplica en las investigaciones relativas al conocimiento indígena y TIC, concibe el contexto determinante para el desarrollo de prototipos tecnológicos en el marco del conocimiento indígena y de sus características, así como de los sujetos que harán uso de los prototipos tecnológicos.

\section{Análisis de datos}

En la revisión bibliográfica, se identificaron 5 dimensiones en las investigaciones o experiencias que abonan en el contexto del conocimiento indígena yTIC, algunasinvestigaciones inciden en más de una dimensión, además de que los trabajos aportan de manera indirecta en alguna de las otras, por lo que fueron los objetivos de las investigaciones los que determinaron la dimensión correspondiente.

\footnotetext{
' Se decidió dejar el concepto en inglés debido a que no se encontró en la bibliografía una traducción al español. Embodied interaction se refiere a una escuela fenomenológica que se fundamenta en la Theory of Embodiment (Dourish, 2004).
} 
http://doi.org/10.15359/ree.24-2.6

ROR: https://ror.org/01t466c14 Universidad Nacional, Costa Rica

http://www.una.ac.cr/educare

educare@una.cr

En la Tabla 1, se muestran los trabajos hallados, se organizan de la siguiente forma: la primera columna corresponde al número de investigación que se le asignó; la segunda columna contiene las abreviaciones para el tipo de fuente, se le asigno A para artículos; C para conferencias; S para simposio y CL para capítulo de libro; en la tercera columna contiene el nombre de los autores y autoras; las siguientes cinco columnas se refieren a las dimensiones.

Tabla 1: Características y elementos de las dimensiones de análisis 4

\begin{tabular}{|c|c|c|c|c|c|c|c|}
\hline \multirow[b]{2}{*}{ 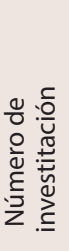 } & \multirow[b]{2}{*}{$\begin{array}{l}\text { Tipo de } \\
\text { fuente }\end{array}$} & \multirow[b]{2}{*}{ Fuente } & \multicolumn{5}{|c|}{ Dimensión en la que intervienen las investigaciones, experiencias e iniciativas revisadas } \\
\hline & & & $\begin{array}{l}\text { Dimensión } 1 \\
\text { Contenido } \\
\text { digital e } \\
\text { inclusión digital }\end{array}$ & $\begin{array}{l}\text { Dimensión } 2 \\
\text { Preservación del } \\
\text { conocimiento }\end{array}$ & $\begin{array}{c}\text { Dimensión } 3 \\
\text { Gestión del } \\
\text { conocimiento }\end{array}$ & $\begin{array}{c}\text { Dimensión } 4 \\
\text { Conocimiento } \\
\text { indígena y TIC } \\
\text { en procesos } \\
\text { educativos }\end{array}$ & $\begin{array}{l}\text { Dimensión } 5 \\
\text { Diseño de } \\
\text { prototipos } \\
\text { contextuales }\end{array}$ \\
\hline 1 & A & $\begin{array}{l}\text { Bidwell, Winschiers-Theophilus, } \\
\text { Koch-Kapuire y Chivuno-Kuria } \\
\text { (2011) }\end{array}$ & & & & & $\bullet$ \\
\hline 2 & A & Zaman et al. (2013) & & & $\bullet$ & & \\
\hline 3 & A & Slavsky (2007) & $\bullet$ & & & & \\
\hline 4 & A & $\begin{array}{l}\text { Bidwell, Winschiers-Theophilus, } \\
\text { Koch-Kapuire y Rehm (2011) }\end{array}$ & & & & & $\bullet$ \\
\hline 5 & A & Reitmaier et al. (2011) & & & & & $\bullet$ \\
\hline 6 & A & Bidwell (2016) & & & & & $\bullet$ \\
\hline 7 & A & Jain (2006) & & $\bullet$ & $\bullet$ & & \\
\hline 8 & A & Sompong y Rampai (2015) & & & $\bullet$ & & \\
\hline 9 & A & Brenes (2012) & $\bullet$ & & & & \\
\hline 10 & A & Asís (2015) & & & & $\bullet$ & \\
\hline 11 & A & Sierra et al. (2010) & & & & $\bullet$ & \\
\hline 12 & C & Thinyane et al. (2007) & $\bullet$ & & $\bullet$ & & \\
\hline 13 & $\mathrm{C}$ & Winchiers-Theophilus et al. (2010) & & & & & - \\
\hline 14 & C & Valbuena (2009) & $\bullet$ & $\bullet$ & & & \\
\hline 15 & C & Maema et al. (2013) & & & & $\bullet$ & \\
\hline 16 & C & Loewen y Dr. Kinshuk (2012) & & & $\bullet$ & $\bullet$ & \\
\hline 17 & C & Bidwell et al. (2010) & & & & & $\bullet$ \\
\hline 18 & $\mathrm{C}$ & Bidwell, Reimaier et al. (2014) & & & & & $\bullet$ \\
\hline
\end{tabular}


http://doi.org/10.15359/ree.24-2.6 ROR: https://ror.org/01t466c14 Universidad Nacional, Costa Rica http://www.una.ac.cr/educare educare@una.cr

\begin{tabular}{|c|c|c|c|c|c|c|c|}
\hline \multirow[b]{2}{*}{ 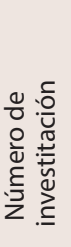 } & \multirow[b]{2}{*}{$\begin{array}{l}\text { Tipo de } \\
\text { fuente }\end{array}$} & \multirow[b]{2}{*}{ Fuente } & \multicolumn{5}{|c|}{ Dimensión en la que intervienen las investigaciones, experiencias e iniciativas revisadas } \\
\hline & & & $\begin{array}{c}\text { Dimensión } 1 \\
\text { Contenido } \\
\text { digital e } \\
\text { inclusión digital }\end{array}$ & $\begin{array}{l}\text { Dimensión } 2 \\
\text { Preservación del } \\
\text { conocimiento }\end{array}$ & $\begin{array}{c}\text { Dimensión } 3 \\
\text { Gestión del } \\
\text { conocimiento }\end{array}$ & $\begin{array}{c}\text { Dimensión } 4 \\
\text { Conocimiento } \\
\text { indígena y TIC } \\
\text { en procesos } \\
\text { educativos }\end{array}$ & $\begin{array}{l}\text { Dimensión } 5 \\
\text { Diseño de } \\
\text { prototipos } \\
\text { contextuales }\end{array}$ \\
\hline 19 & C & Bidwell et al. (2008) & & & & & $\bullet$ \\
\hline 20 & C & Khalala et al. (2014) & & $\bullet$ & & & \\
\hline 21 & C & Reitsma et al. (2013) & & $\bullet$ & & & \\
\hline 22 & C & Zaman et al. (2015) & & $\bullet$ & & & \\
\hline 23 & C & Awori et al. (2015) & & & & & $\bullet$ \\
\hline 24 & S & Thapa y Devi (2015) & & $\bullet$ & & & \\
\hline 25 & $\mathrm{CL}$ & Siew et al. (2013) & & & $\bullet$ & & \\
\hline 26 & $\mathrm{CL}$ & McNulty (2014) & & & $\bullet$ & & \\
\hline 27 & $\mathrm{CL}$ & Rodil et al. (2011) & & & & & $\bullet$ \\
\hline & & Total & 4 & 6 & 7 & 4 & 10 \\
\hline & & Porcentaje & $12.9 \%$ & $19.3 \%$ & $22.5 \%$ & $12.9 \%$ & $32.2 \%$ \\
\hline
\end{tabular}

Nota: Elaboración propia.

De acuerdo con los porcentajes mostrados en la Tabla 1, es posible observar que la mayoría de los trabajos se encuentran en lo referido al diseo de prototipos contextuales con un $32.3 \%$; en segundo lugar aparece la gestión del conocimiento con un $22.5 \%$; los referidos a la preservación del conocimiento tienen un $19.3 \%$, las dimensiones creación de contenido digital e inclusión digital y conocimiento indígena y TIC en procesos educativos tienen un $12.9 \%$. No obstante, de acuerdo con las fechas de publicación de las investigaciones, es posible inferir que la dimensión conocimiento indígena TIC en procesos educativos se perfila como una línea de investigación en fortalecimiento.

\section{Dimensión 1: Creación de contenido digital cultural e inclusión digital}

Esta dimensión agrupa las categorías: creación de contenido digital; apropiación tecnológica; inclusión digital. Las investigaciones abordan de manera indirecta la apropiación tecnológica y esta es requisito indispensable para el abordaje de la creación de contenido digital cultural. Estas creaciones de contenido digital son, en su mayoría ,grabaciones de audio y vídeo, y abonan en la preservación de la lengua, la Tabla 2 muestra los trabajos hallados. 
http://doi.org/10.15359/ree.24-2.6

ROR: https://ror.org/01t466c14 Universidad Nacional, Costa Rica

http://www.una.ac.cr/educare

educare@una.cr

Tabla 2: Experiencias en el marco de la creación de contenidos culturales digitales e inclusión digital

\begin{tabular}{|c|c|c|}
\hline Título & Autoría & Aportación \\
\hline $\begin{array}{l}\text { TIC para jóvenes indígenas: Una experiencia } \\
\text { mapuche en la creación colectiva de } \\
\text { contenidos culturales digitales }\end{array}$ & (Slavsky, 2007) & $\begin{array}{l}\text { - Inclusión digital } \\
\text { - Acceso a la sociedad del conocimiento } \\
\text { - Plataformas virtuales libres }\end{array}$ \\
\hline $\begin{array}{l}\text { Multimedia como estrategia para la } \\
\text { introducción de TIC en las comunidades } \\
\text { indígenas cabécares de Chirripó }\end{array}$ & (Brenes, 2012) & $\begin{array}{l}\text { - Inclusión digital } \\
\text { - Apropiación TIC } \\
\text { - Preservación de la lengua }\end{array}$ \\
\hline $\begin{array}{l}\text { An ontology-based, multi-modal platform } \\
\text { for the inclusion of marginalized rural } \\
\text { communities into the knowledge society }\end{array}$ & $\begin{array}{l}\text { (Thinyane et al., } \\
\text { 2007) }\end{array}$ & $\begin{array}{l}\text { - Inclusión digital } \\
\text { - Plataforma sensible al conocimiento indígena }\end{array}$ \\
\hline $\begin{array}{l}\text { Digital inclusion of indigenous people } \\
\text { in Colombia, by the digitalization and } \\
\text { safeguarding of their intangible cultural } \\
\text { Hheritage }\end{array}$ & (Valbuena, 2009) & $\begin{array}{l}\text { - Inclusión digital } \\
\text { - Digitalización de patrimonio cultural } \\
\text { - Mejorar la calidad de las comunidades indígenas } \\
\text { - Conservación del patrimonio cultural intangible }\end{array}$ \\
\hline
\end{tabular}

Nota: Elaboración propia.

Estas aproximaciones se hicieron con el objetivo de acercar a los grupos indígenas a la era digital o para introducir las TIC a las comunidades, como estrategia para lograrlo se hace a través de la cultura, en particular lo relativo a la lengua e historias que los pueblos guardan dentro de su patrimonio cultural.

En los estudios de Alamo (2003), Hernández y Calcagno (2003, citados en Slavsky, 2007) se menciona que la implementación de programas de acceso digital debe ser el resultado de la participación de las comunidades indígenas y [debe ser] respetuosa de las estructuras sociales, culturales y económicas y que promuevan la gestión autónoma de las comunidades (Slavsky, 2007). Un aspecto que se debe considerar, de acuerdo con Castells (2002), es que, si bien las TIC no son malas, tampoco son necesariamente buenas e, incluso, tampoco neutras, sino que dependen de sus usos, por lo que considerar la implementación de programas debe hacerse desde perspectivas socioculturales propias. En ese sentido, los trabajos presentados en esta dimensión son el resultado de consultas con la comunidad respecto al tipo de contenido cultural.

\section{Dimensión 2: Preservación de conocimiento indígena}

Digitalización del conocimiento indígena, conocimiento en formato y repositorios digitales son las categorías de la dimensión 2. El propósito de los trabajos es la preservación de conocimiento indígena, sin embargo, algunos también mencionan entre sus propósitos 
http://doi.org/10.15359/ree.24-2.6

ROR: https://ror.org/01t466c14 Universidad Nacional, Costa Rica

la gestión del conocimiento. La Tabla 3 muestran los trabajos analizados y sus resultados correspondientes a la preservación del conocimiento indígena, en los artículos, se incluyen revisiones bibliográficas, en tanto que hacen recomendaciones para la preservación del conocimiento indígena en el contexto africano e hindú.

Tabla 3: Investigaciones que abonan en la preservación del conocimiento indígena

\begin{tabular}{|c|c|c|}
\hline Título & Autoría & Resultados \\
\hline $\begin{array}{l}\text { Empowering Africa's development } \\
\text { using ICT in a knowledge } \\
\text { management approach }\end{array}$ & (Jain, 2006) & $\begin{array}{l}\text { - Recomendaciones para usar las TIC en la gestión } \\
\text { y preservación del conocimiento indígena }\end{array}$ \\
\hline $\begin{array}{l}\text { A case for understanding user } \\
\text { experience challenges confronting } \\
\text { indigenous knowledge recorders in } \\
\text { ruralcommunities in South Africa }\end{array}$ & (Khalala et al., 2014) & $\begin{array}{l}\text { - Se hace énfasis en el proceso y las herramientas } \\
\text { de recolección del conocimiento indígena } \\
\text { así como su almacenamiento es crítico en su } \\
\text { calidad. } \\
\text { - Se realiza un estudio de la experiencia de sujeto } \\
\text { usuario para generar recomendaciones en el } \\
\text { proyecto. }\end{array}$ \\
\hline $\begin{array}{l}\text { StoryBeads: Preserving indigenous } \\
\text { knowledge through tangible } \\
\text { interaction design }\end{array}$ & (Reitsma et al., 2013) & $\begin{array}{l}\text { - Diseño del prototipo TIC centrado en el público } \\
\text { usuario. } \\
\text { - Se siguió un proceso iterativo para entender la } \\
\text { cultura y diseñar un prototipo culturalmente } \\
\text { relevante. }\end{array}$ \\
\hline $\begin{array}{l}\text { Reviving an indigenous rainforest } \\
\text { sign language: Digital Oroo' } \\
\text { adventure game }\end{array}$ & (Zaman, et al. 2015) & $\begin{array}{l}\text { - Despierta el interés de la niñez en videojuegos } \\
\text { de contenido indígena. } \\
\text { - Diseño que mide usabilidad }\end{array}$ \\
\hline $\begin{array}{l}\text { Preservation of the traditional } \\
\text { knowledge of tribal population in India }\end{array}$ & (Thapa y Devi , 2015) & $\begin{array}{l}\text { - Se analizan las iniciativas gubernamentales de } \\
\text { India } \\
\text { - El estudio muestra que los esfuerzos están } \\
\text { dispersos, hace falta una política concreta. }\end{array}$ \\
\hline
\end{tabular}

Nota: Elaboración propia.

Es posible observar la introducción de las TIC en la preservación del conocimiento a través de videojuegos (Zaman et al., 2015) y diseños de prototipos que toman en consideración la cultura (Reitsma et al., 2013). Además, se estudia el caso de las iniciativas de India (Thapa y Devi, 2015), y se concluye que hace falta una política concreta. 
http://doi.org/10.15359/ree.24-2.6

ROR: https://ror.org/01t466c14 Universidad Nacional, Costa Rica

http://www.una.ac.cr/educare

educare@una.cr

\section{Dimensión 3. La gestión del conocimiento indígena}

Las categorías de la gestión del conocimiento son repositorios digitales y empoderamiento de la comunidad. Antes de iniciar con el análisis, se exponen algunas definiciones relativas a la gestión del conocimiento.

La gestión del conocimiento es un concepto que tiene diversas definiciones, por ejemplo, Skyrme (2001, citado en Jain, 2006), lo define como "a process or practice of creating, acquiring, capturing, sharing and using knowledge, wherever it resides, to enhance learning and performance in organizations" p. 2. De acuerdo con Mendoza (2011), existen dos áreas en la gestión del conocimiento, una dedicada propiamente a la gestión del conocimiento que surge por la necesidad de valorar los recursos intangibles de las empresas y otra área denominada conocimiento para el desarrollo, que abarca temas de pobreza, desigualdad y crecimiento. Concibe el conocimiento como el conductor del crecimiento. Esta clasificación es de utilidad para describir la gestión del conocimiento indígena en tanto que es puesto a disposición de la comunidad a través de repositorios digitales para empoderarla.

El documento presentado por Jain (2006) hace una serie de recomendaciones para la gestión del conocimiento indígena en particular en Sudáfrica. Zaman et al. (2013) en Malasia (Sompong y Rampai, 2015) realizan un análisis respecto a los centros comunitarios de aprendizaje en Tailandia, en su trabajo lograron identificar que las políticas implementadas para la recolección del conocimiento indígena son insuficientes, en tanto no existe un proyecto a largo plazo.

En la investigación de Siew et al. (2013), se desarrolla el software eTORO, dicho software es producto de la participación tanto del equipo de investigación como de miembros de la comunidad de Long Lamai, Sarawak en Malasia. Este trabajo es un ejemplo de que las dimensiones encontradas en las investigaciones no se limitan a una sola, ya que menciona que uno de los objetivos es la preservación del conocimiento indígena en tanto que la generación adulta está muriendo poco a poco y los conocimientos con ella, de esta forma el conocimiento no está siendo transmitido a las generaciones más jóvenes, por otro lado, la juventud no está interesada en el aprendizaje y preservación del conocimiento indígena.

El estudio de caso realizado por McNulty (2014) analiza el programa Ulwazi, iniciativa que tiene entre sus objetivos la preservación y diseminación del conocimiento indígena. Establecida en 2008 en Sudáfrica, Ulwazi opera como una parte integral de las librerías públicas y servicios de información. La recolección del conocimiento indígena es realizada por personal voluntario, que ha sido capacitado en la recolección. Sus características son, entre otras: ser jóvenes con ciertas habilidades para las TIC, tener cierto grado de confianza con los miembros de la comunidad, reconocer el valor de las personas ancianas y el conocimiento que poseen; sin embargo, una de las limitantes del programa es la movilidad de estos grupos voluntarios 
http://doi.org/10.15359/ree.24-2.6

ROR: https://ror.org/01t466c14 Universidad Nacional, Costa Rica

que continuamente abandonan el programa debido a su necesidad de trabajar. Estos grupos recolectan el conocimiento indígena a través de entrevistas y estas son grabadas en audio y vídeo. Lo anteriormente mencionado se muestra en la Tabla 4.

Tabla 4: Aproximaciones investigativas en el marco de la gestión del conocimiento indígena

\begin{tabular}{|c|c|c|}
\hline Título & Autores & Resultados \\
\hline $\begin{array}{l}\text { Empowering Africa's development } \\
\text { using ICT in a knowledge } \\
\text { management approach }\end{array}$ & (Jain, 2006) & $\begin{array}{l}\text { - Recomendaciones para usar las TIC en la } \\
\text { gestión y preservación del conocimiento } \\
\text { indígena. }\end{array}$ \\
\hline $\begin{array}{l}\text { Augmenting indigenous knowledge } \\
\text { management with information and } \\
\text { communication technology }\end{array}$ & (Zaman et al., 2013) & $\begin{array}{l}\text { - Diseño iterativo } \\
\text { - Se usa un sistema de gestión de } \\
\text { contenido. } \\
\text { - Lograron que jóvenes interactuaran más } \\
\text { con los abuelos sabios }\end{array}$ \\
\hline $\begin{array}{l}\text { The development model of } \\
\text { knowledge management to } \\
\text { strengthen Thai ict community } \\
\text { learning center }\end{array}$ & (Sompong y Rampai, 2015) & $\begin{array}{l}\text {-Encuentran que no existen actividades } \\
\text { planeadas a largo plazo ni de } \\
\text { mantenimiento en el estudio y colección } \\
\text { del conocimiento indígena. }\end{array}$ \\
\hline $\begin{array}{l}\text { Participatory action research in } \\
\text { software development: Indigenous } \\
\text { knowledge management systems } \\
\text { case study }\end{array}$ & (Siew et al., 2013) & $\begin{array}{l}\text { - Desarrollo de una plataforma para la } \\
\text { gestión del Cl } \\
\text { - La plataforma es valorada por miembros } \\
\text { de la comunidad en cuestión }\end{array}$ \\
\hline $\begin{array}{l}\text { The Ulwazi programme: A case study } \\
\text { in community-focused indigenous } \\
\text { knowledge management }\end{array}$ & (McNulty, 2014) & $\begin{array}{l}\text { - La política de la iniciativa permite que } \\
\text { la gente digitalice lo que cree que es } \\
\text { importante para que tenga sentido y } \\
\text { lógica para la población } \\
\text { - La mayoría del público usuario de la } \\
\text { plataforma son habitantes de lengua Zulu }\end{array}$ \\
\hline
\end{tabular}

Nota: Elaboración propia.

Los resultados de las investigaciones vierten recomendaciones en torno a la gestión de los sistemas de conocimiento, así mismo es posible notar que el tema es de interés de los gobiernos, ya que se pudieron hallar iniciativas en esta dimensión. Es de notar que los nativos son quienes recolectan el conocimiento y se respeta el tipo de conocimiento que las comunidades desean colocar en las plataformas virtuales. 
http://doi.org/10.15359/ree.24-2.6

ROR: https://ror.org/01t466c14 Universidad Nacional, Costa Rica

http://www.una.ac.cr/educare

educare@una.cr

\section{Dimensión 4. Conocimiento indígena y TIC en procesos educativos}

El reconocimiento del conocimiento indígena para usarse en contextos educativos, el diseño de plataformas individuales para acceder al conocimiento indígena, así como el desarrollo de objetos de aprendizaje con base en el conocimiento y el contexto indígena son las categorías de esta dimensión. La incursión de las TIC en el contexto educativo es un tópico que ha generado multitud de investigaciones, sin embargo, el tema del conocimiento indígena y las TIC en los procesos educativos es un tema poco abordado. Algunos países, como Canadá, han empezado a retomar el conocimiento indígena para incluirlo en los contextos educativos formales (Cházaro, 2014). En la Tabla 5 se muestran algunos estudios relacionados con esta dimensión.

Tabla 5: Propuestas relativas a conocimiento indígena y TIC en procesos educativos

\begin{tabular}{|c|c|c|}
\hline Título & Autoría & Resultados \\
\hline $\begin{array}{l}\text { A look into classification: } \\
\text { Towards buiding an indigenous } \\
\text { knowledege platform for } \\
\text { educational use }\end{array}$ & (Maema et al., 2013) & $\begin{array}{l}\text { - Recomendaciones para usar el } \\
\text { conocimiento indígena en sistemas } \\
\text { formales de educación }\end{array}$ \\
\hline $\begin{array}{l}\text { The need for technological } \\
\text { innovations for indigenous } \\
\text { knowledge transfer in culturally } \\
\text { inclusive education }\end{array}$ & (Loewen y Dr. Kinshuk, 2012) & $\begin{array}{l}\text { - Plataforma de gestión del conocimiento } \\
\text { indígena disponible para su uso en } \\
\text { contextos educativos formales } \\
\text { - Metodología de diseño participativo } \\
\text { - Creación de objetos de aprendizaje }\end{array}$ \\
\hline $\begin{array}{l}\text { Una comunidad virtual de apoyo } \\
\text { a procesos de etnoeducación } \\
\text { para la comunidad nasa } \\
\text { del resguardo de Corinto } \\
\text { Lópezadentro, departamento del } \\
\text { Cauca-Colombia }\end{array}$ & (Sierra et al., 2010) & $\begin{array}{l}\text { - Incorporación de las TIC en la dinámica de } \\
\text { los procesos de etnoeducación } \\
\text { - Comunidad virtual centrada en los } \\
\text { elementos culturales de la comunidad } \\
\text { - Comunidad virtual con servicios que la } \\
\text { soportan con respeto a la cosmovisión de la } \\
\text { cultura nasa }\end{array}$ \\
\hline $\begin{array}{l}\text { Las TIC y su inmersión en los } \\
\text { proyectos etnoeducativos de las } \\
\text { comunidades indígenas }\end{array}$ & (Asís, 2015) & $\begin{array}{l}\text { - Estudio que abarca diferentes comunidades } \\
\text { indígenas. } \\
\text { - Se establece que las TIC fortalecen la cultura } \\
\text { y empoderan a las comunidades. }\end{array}$ \\
\hline
\end{tabular}

Nota: Elaboración propia.

Los trabajos de la presente dimensión inciden también en la gestión del conocimiento indígena, ya que, para incluirlo en la educación formal, se busca recolectarlo y, posteriormente, colocarlo en una plataforma virtual, e incluso se propone el diseño de objetos de aprendizaje. 
http://doi.org/10.15359/ree.24-2.6

ROR: https://ror.org/01t466c14 Universidad Nacional, Costa Rica

\section{Dimensión 5. Diseño de prototipos tecnológicos acordes con el contexto indígena}

Las categorías que comprenden esta dimensión son diseño de prototipos centrados en el público usuario y diseños iterativos; en esta dimensión, las investigaciones se enfocan en el diseño de prototipos tecnológicos sensibles a las características del conocimiento indígena. Los diseños generalmente son realizados en colaboración con las comunidades de estudio y son el resultado de procesos iterativos mediante los cuales es posible adaptar los prototipos a las necesidades de la comunidad en cuestión, por lo cual derivan en diseños culturalmente relevantes. Las aproximaciones investigativas usan, en su mayoría, diseños metodológicos críticos, en particular la investigación-acción, aunque entre sus resultados se menciona que no se ha llegado a un punto en el cual los diseños de los prototipos sean, en su totalidad, sensibles a las características del conocimiento indígena ni a la lógica cultural de las comunidades (Bidwell, Winschiers-Theophilus, Koch-Kapuire y Rehm, 2011; Rodil et al., 2011).

Por su parte Bidwell (2016) propone el diseño de prototipos desde una perspectiva de-colonial en tanto que, al tenerse una formación opuesta a los sistemas de conocimiento indígena y completamente occidental, los diseños suelen estar permeados en su totalidad de esta formación, lo que limita la propuesta de diseños más apegados al contexto indígena. Las investigaciones y sus principales aportaciones se muestran en la Tabla 6.

Tabla 6: Investigaciones sobre diseño de prototipos tecnológicos acordes con el contexto del conocimiento indígena

\begin{tabular}{|c|c|c|}
\hline Título & Autoría & Resultados \\
\hline $\begin{array}{l}\text { Situated interactions between } \\
\text { audiovisual media and African } \\
\text { herbal lore }\end{array}$ & $\begin{array}{l}\text { (Bidwell, Winschiers- } \\
\text { Theophilus, Koch-Kapuire y } \\
\text { Chivuno Kuria, 2011) }\end{array}$ & $\begin{array}{l}\text { - Sus hallazgos proponen nuevos patrones de diseño para orientar la } \\
\text { representación de la herbolaria dentro de espacios que contextualicen el } \\
\text { conocer hacer y los movimientos. } \\
\text { - Los vídeos fueron capaces de capturar parte importante de la pedagogía } \\
\text { de la transmisión del conocimiento herbolario. }\end{array}$ \\
\hline $\begin{array}{l}\text { Pushing personhood into } \\
\text { pace: Situating media in rural } \\
\text { knowledge in Africa }\end{array}$ & $\begin{array}{l}\text { (Bidwell, Winschiers- } \\
\text { Theophilus, Koch-Kapuire y } \\
\text { Rehm, 2011) }\end{array}$ & $\begin{array}{l}\text { - El movimiento corporal juega un papel importante en el conocimiento, } \\
\text { se deben mejorar las TIC para ampliar las prácticas de conocimiento, } \\
\text { para dar respuesta a las interconexiones de factores sociales, espaciales y } \\
\text { temporales, los que producen pautas. }\end{array}$ \\
\hline $\begin{array}{l}\text { Situating digital storytelling } \\
\text { within African communities }\end{array}$ & (Reitmaier et al., 2011) & $\begin{array}{l}\text { - Se creó un prototipo más adecuado a las necesidades de la comunidad y } \\
\text { a la oralidad. } \\
\text { - Refieren que la investigación está influenciada por su bagaje cultural, y } \\
\text { este es el reto más grande para el diseño en culturas no occidentales. }\end{array}$ \\
\hline $\begin{array}{l}\text { Moving the centre to design } \\
\text { social media in rural Africa }\end{array}$ & (Bidwell, 2016) & $\begin{array}{l}\text { - Propone diseños de prototipos tecnológicos de-coloniales. } \\
\text { - El acceso y tipo de lo que se graba y comparte depende de la jefatura } \\
\text { - Por otro lado, sugieren que la grabación y escuchar archivos de voz en } \\
\text { un dispositivo de pantalla táctil de propiedad comunal. }\end{array}$ \\
\hline $\begin{array}{l}\text { Determining requirements } \\
\text { within an indigenous } \\
\text { knowledge system of African } \\
\text { rural communities }\end{array}$ & $\begin{array}{l}\text { (Winschiers-Theophilus et al. } \\
\text { (2010) }\end{array}$ & $\begin{array}{l}\text { - Manifiestan en sus resultados el respeto a la lógica cultural, valores } \\
\text { sociales y espirituales de las comunidades indígenas. } \\
\text { - Los prejuicios propios ontológicos y de representación pueden limitar la } \\
\text { obtención de los significados locales. }\end{array}$ \\
\hline
\end{tabular}


http://doi.org/10.15359/ree.24-2.6

ROR: https://ror.org/01t466c14 Universidad Nacional, Costa Rica

http://www.una.ac.cr/educare

educare@una.cr

\begin{tabular}{|c|c|c|}
\hline Título & Autoría & Resultados \\
\hline $\begin{array}{l}\text { Designing with mobile digital } \\
\text { storytelling in rural Africa }\end{array}$ & (Bidwell et al., 2010) & $\begin{array}{l}\text { - Desarrollan una aplicación para la creación de relatos digitales con base } \\
\text { en la lógica cultural. } \\
\text { - Idearon un método para explorar la narrativa digital de una manera más } \\
\text { matizada. } \\
\text { - El prototipo es de alguna forma un diseño que permite al público usuario } \\
\text { expresarse por sí mismo y esta será clave en el diseño de trabajos futuros }\end{array}$ \\
\hline $\begin{array}{l}\text { Designing social media for } \\
\text { community information } \\
\text { sharing in rural South Africa }\end{array}$ & (Bidwell, Robinson, 2014) & $\begin{array}{l}\text { - El diseño se centra en las prácticas de comunicación intergeneracional. } \\
\text { - Se reflexiona acerca del cómo usar prototipos que consideren las } \\
\text { prácticas de comunicación intergeneracional y generacional. }\end{array}$ \\
\hline $\begin{array}{l}\text { The landscape's apprentice: } \\
\text { Lessons for place-centred } \\
\text { design from grounding } \\
\text { documentary }\end{array}$ & (Bidwell et al., 2008) & $\begin{array}{l}\text { - Concluyen que la tecnología en contextos no urbanos debe tener un } \\
\text { enfoque menos antropocéntrico. } \\
\text { - Se exhorta a los equipos diseñadores a usar una espacialidad diferente a } \\
\text { la cartesiana. } \\
\text { - Se critica el espacio y temporalidad en muchos métodos de video } \\
\text { utilizados en el análisis y diseño para enfatizar el valor de conservar la } \\
\text { integridad de la transferencia de conocimientos. }\end{array}$ \\
\hline $\begin{array}{l}\text { Transnationalism, indigenous } \\
\text { knowledge and technology: } \\
\text { linsights from the Kenyan } \\
\text { diaspora }\end{array}$ & (Awori et al., 2015) & $\begin{array}{l}\text { - Encontraron un interés inesperado en jóvenes por el conocimiento } \\
\text { indígena } \\
\text { - Se hace hincapié en más que la preservación el cultivo del conocimiento } \\
\text { indígena. }\end{array}$ \\
\hline $\begin{array}{l}\text { A new visualization approach } \\
\text { to re-ccontextualize } \\
\text { indigenous knowledge in rural } \\
\text { Africa }\end{array}$ & (Rodil et al., 2011) & $\begin{array}{l}\text { - Se debe tomar en cuenta las trasformaciones que tienen lugar cuando } \\
\text { la tecnología interactúa en las experiencias de vida, acciones y } \\
\text { pensamiento o narrativa que constituyen el conocimiento indígena. } \\
\text { - El prototipo diseñado es un buen intento para re-contextualizar } \\
\text { representaciones descontextualizadas de una manera localmente accesible. }\end{array}$ \\
\hline
\end{tabular}

Nota: Elaboración propia.

El tipo de tecnología que se diseña o propone en esta dimensión son aplicaciones para realizar relatos digitales; hacen alusión a la oralidad, social media, es decir, espacios para compartir conocimiento y audio-repositorios (Bidwell, 2016; Bidwell et al., 2010; Bidwell, Robinson et al., 2014). Un resultado importante que se debe resaltar en esta dimensión es que los dispositivos están diseñados para que tengan un uso individual, mientras que en los contextos indígenas se usan de manera colectiva.

El autor más mencionado, en nueve de los diez trabajos, es Nicola Bidwell, y el país que más trabaja este tipo de investigaciones es Sudáfrica. Aunque Bidwell, no es la única autora que aborda este tipo de investigaciones, otros autores reiterativos son Heike Winschiers-Teophilus, Kasper Rodil y Gereon Koch Kapuire. Tales profesionales trabajan en la línea de investigación de $\mathrm{HCl}$, y el tercer paradigma emergente es su línea de investigación actual.

\section{Conclusiones}

En la revisión de la bibliografía fue posible identificar diversas investigaciones, experiencias, iniciativas gubernamentales y revisiones de textos e investigaciones que abonan al tema del conocimiento indígena y las TIC, así mismo se pudo identificar que estas se encuentran distribuidas en cinco dimensiones. 
En la dimensión inclusión digital y creación de contenido digital cultural, las investigaciones se encontraron en su mayoría en Latinoamérica (Brenes, 2012; Slavsky, 2007; Valbuena, 2009), y una más en Sudáfrica (Thinyane et al., 2007); sin embargo, estas son un número reducido en comparación con las otras cuatro dimensiones y las pocas que se hallaron se han publicado ya hace tiempo (2007, 2009 y 2012). Entre los resultados o aportaciones de las investigaciones, se encontró que los grupos indígenas no son ajenos a las TIC y que solo las usan si estas adquieren sentido para ellos (Slavsky, 2007), también se encontró que les dan sentido a las TIC cuando estas las usan para difundir su cultura (Brenes, 2012); sin embargo, existe un vacío en las investigaciones con respecto a las generaciones actuales y el sentido que tiene para ellas el uso de las TIC. A medida que transcurre el tiempo, los avances tecnológicos y su penetración en diversos grupos culturales es cada vez mayor, por lo que habría que indagar si los grupos indígenas están interesados en usar las TIC sí y solo sí difunden a través de ellas su cultura. O quizá ya han penetrado en sus contextos al punto que las usan como cualquier otro grupo cultural que tenga acceso al internet y ciertas habilidades con las TIC para informarse y usar esa nueva información, o incluso si la penetración es tal que ya forman parte de los procesos y acciones propias de la ciudadanía digital (pagan impuestos, hacen transferencias, revisan plataformas políticas, se enteran de las noticias, buscan información para sus actividades económicas, etc.).

En la dimensión preservación del conocimiento indígena, Reitsma et al. (2013) usan procesos iterativos para el diseño de prototipos hasta lograr contextualizar, en mayor medida, sus propuestas. Zaman et al. (2013) y Zaman et al. (2015) indican una ruptura intergeneracional, lo que contribuye a la erosión del conocimiento así como a la necesidad de generar propuestas culturalmente relevantes. Hasta el momento no se han encontrado investigaciones que den cuenta de los resultados a largo plazo, en particular para indagar acerca de la pertinencia de las propuestas.

La gestión del conocimiento indígena mediada por las TIC tiene como objetivo empoderara la comunidad a través de su conocimiento. Una problemática mencionada acerca de las iniciativas gubernamentales es que no hay implementados proyectos de gestión del conocimiento a largo plazo (Sompong y Rampai, 2015). En la revisión, siguiendo la búsqueda y selección indicada al inicio de este capítulo, no se hallaron investigaciones que hayan estudiado el funcionamiento a largo plazo de las propuestas, por lo que sería interesante descubrir hasta qué punto es posible gestionar el conocimiento indígena de manera pertinente para las comunidades indígenas.

Respecto a la inclusión del conocimiento indígena y las TIC en contextos formales educativos, las investigaciones no son tan vastas en comparación con otras dimensiones. Se encontraron trabajos realizados principalmente en Colombia y Canadá, los resultados presentados muestran que la cultura se fortalece y las comunidades se empoderan (Asís, 2015; Sierra et al., 2010), así mismo, se evidencia que las propuestas educativas son respetuosas de la comunidad en cuestión, también se encuentra la creación de objetos de aprendizaje. De manera general, el campo ha sido poco explorado, por lo que se podría abordar en investigaciones que incidan en la inmersión del conocimiento indígena en el contexto formal educativo, no obstante, en lo que se refiere a objetos de aprendizaje se manifiesta una prolífera línea de investigación. 
http://doi.org/10.15359/ree.24-2.6

ROR: https://ror.org/01t466c14 Universidad Nacional, Costa Rica

http://www.una.ac.cr/educare

educare@una.cr

El diseño de prototipos tecnológicos acordes al contexto indígena utiliza básicamente investigación de corte etnográfico. En esta dimensión, las investigaciones reconocen que, para el diseño de sus propuestas, es necesario tomar en cuenta la pedagogía de trasmisión del conocimiento indígena, reconocen su característica oral, así como la importancia del lenguaje corporal; por otro lado, dan cuenta de las limitaciones que tienen las TIC para adecuarse a contextos indígenas (Bidwell, Winschiers-Theophilus, Koch-Kapuire y Rehm, 2011; Bidwell, Reitmaier et al., 2014; Rodil et al., 2011). En esta dimensión, se proponen prototipos cuyo diseño es el resultado de procesos iterativos, sin embargo, un espacio de investigación que puede generarse es el uso de recursos tecnológicos existentes que vayan con la lógica del software libre en tanto que las comunidades indígenas se encuentran en sintonía con esta mentalidad. De manera general, en las cinco dimensiones, existe un vacío en la bibliografía generada en países como México, por lo que abordar este tipo de temas resulta necesario, sobre todo considerando la característica multicultural de este país.

En la Figura 1 se representa gráficamente la dimensión “diseño de prototipos tecnológicos acordes al contexto indígena", considerada la más relevante en tanto que aglutina y se puede conformar por tres dimensiones: Preservación del conocimiento; gestión del conocimiento; y creación de contenido digital cultural, e inclusión cultural.

Figura 1: Dimensión diseño de prototipos tecnológicos acordes al contexto indígena

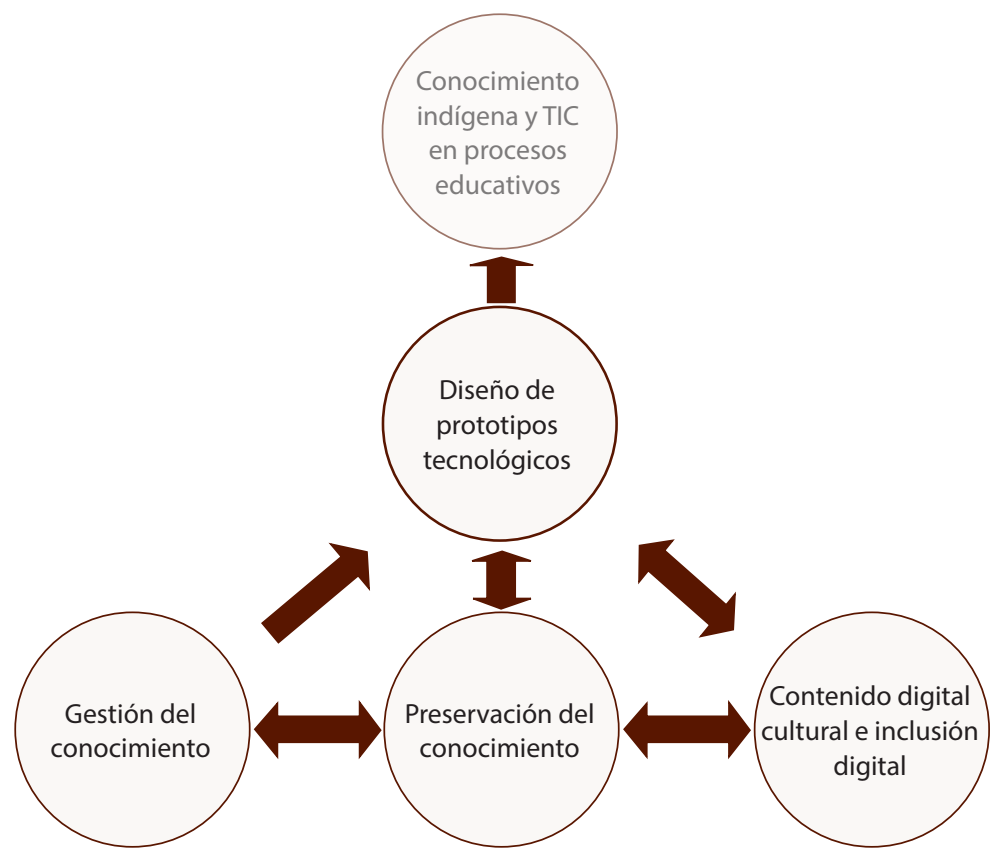

Nota: Elaboración propia. 
A partir del análisis realizado en la revisión bibliográfica, se identifica que las tres dimensiones proveen de elementos adecuados para la promoción y preservación del conocimiento indígena en el ámbito educativo formal, con el uso de las TIC. Estas últimas, identificadas como herramientas que además de potenciar la preservación incorporan a las poblaciones indígenas al mundo digital.

Una posible área en desarrollo es la conjunción de la gestión, educación y TIC centrada en la preservación del conocimiento indígena, lo que a su vez podría empoderar a las comunidades en tanto que sean ellas las que trabajen en las propuestas.

El análisis también ofrece elementos para identificar un tema o área del conocimiento con amplio potencial para el desarrollo de investigaciones del tipo multi y transdisciplinar, particularmente para México.

Finalmente, al análisis permitió notar la ausencia del entendimiento a priori de las epistemologías nativas para transmitir lo más fielmente posible el conocimiento por medio de tecnologías digitales, al mismo tiempo que permita comprender el significado y la importancia que cada pueblo indígena le proporciona al conocimiento indígena.

\section{Referencias}

Asís, R. (2015). Las TIC y su inmersión en los proyectos etnoeducativos de las comunidades indígenas. En J. C. Arboleta (Ed.), Dispositivos para el aprendizaje y formación (Tomo 20, pp. 165-182). Redilpe.

Awori, K., Vetere, F. y Smith, W. (2015). Transnationalism, indigenous knowledge and technology: Insights from the Kenyan diaspora. En Proceedings of the 33rd Annual ACM Conference on Human Factors in Computing Systems (pp. 3759-3768). ACM. https://doi. org/10.1145/2702123.2702488

Bidwell, N. J. (2016). Moving the centre to design social media in rural Africa. Al \& Society, 31(1), 51-77. https://doi.org/10.1007/s00146-014-0564-5

Bidwell, N. J., Reitmaier, T. y Jampo, K. (2014). Orality, gender and social audio in rural Africa. En Rossitto. C., Ciolfi, L., Martin, D. y Conein, B. (Eds), COOP 2014 - Proceedings of the 11th International Conference on the Design of Cooperative Systems, 27-30 May 2014, Nice (France) (pp. 225-242). Springer. https://doi.org/10.1007/978-3-319-06498-7

Bidwell, N. J., Reitmaier, T., Marsden, G. y Hansen, S. (2010). Designing with mobile digital storytelling in rural Africa. En Proceedings of the SIGCHI Conference on Human Factors in Computing Systems (pp. 1593-1602). ACM. https://doi.org/10.1145/1753326.1753564 
http://doi.org/10.15359/ree.24-2.6

ROR: https://ror.org/01t466c14 Universidad Nacional, Costa Rica

http://www.una.ac.cr/educare

educare@una.cr

Bidwell, N. J., Robinson, S., Vartiainen, E., Jones, M., Siya, M. J., Reitmaier, T., ... Lalmas, M. (2014). Designing social media for community information sharing in rural South Africa. En Proceedings of the Southern African Institute for Computer Scientist and Information Technologists Annual Conference 2014 on SAICSIT 2014 Empowered by Technology (pp. 104114). ACM. https://doi.org/10.1145/2664591.2664615

Bidwell, N. J., Standley, P.-M., George, T. y Steffensen, V. (2008). The landscape's apprentice: lessons for place-centred design from grounding documentary. En Proceedings of the 7th ACM Conference on Designing Interactive Systems (pp. 88-98). ACM. https://doi. org/10.1145/1394445.1394455

Bidwell, N. J., Winschiers-Theophilus, H., Koch-Kapuire, G. y Chivuno-Kuria, S. (2011). Situated interactions between audiovisual media and African herbal lore. Personal and Ubiquitous Computing, 15(6), 609-627. https://doi.org/10.1007/s00779-010-0337-1

Bidwell, N. J., Winschiers-Theophilus, H., Koch-Kapuire, G.y Rehm, M. (2011). Pushing personhood into place: Situating media in rural knowledge in Africa. International Journal of Human Computer Studies, 69(10), 618-631. https://doi.org/10.1016/j.ijhcs.2011.02.002

Brenes, C. (2012). Multimedia como estrategia para la introducción de TIC en las comunidades indígenas Cabécares de Chirripó. InterSedes, 13(25), 68-81. http://revistas.ucr.ac.cr/index. php/intersedes/article/view/1990

Castells, M. (2002). La era de la información. Economía, sociedad y cultura (Vol. 1: La sociedad Red). Siglo XXI.

Cházaro, E. (2014). Integración del modelo pedagógico basado en la sabiduria nativa, Cerrando el círculo de aprendizaje (Vol. 2: Hacia la comprensión del paradigma nativo como base epistemológica en la sistematización del conocimiento). Benemérita Universidad Autónoma de Puebla.

Comisión Nacional para el Conocimiento y Uso de la Biodiversidad (CONABIO). (2008). Conocimiento tradicional. https://www.biodiversidad.gob.mx/usos/conotrad.html

Dourish, P. (2004). Where the action is: The foundations of embodied interactions. MIT Press.

Gómez, C. L. (2005). Tejiendo hilos de comunicación: Los usos sociales del internet en los pueblos indígenas de México (Tesis de doctorado). Universidad Nacional Autónoma de México. https://ccdoc.iteso.mx/acervo/cat.aspx?cmn=browse\&id=5115

Harrison, S., Tatar, D. y Sengers, P. (2007). The three paradigms of $\mathrm{HCl}$. HCl, 1-21. https:// pdfs.semanticscholar.org/4a97/a948bbf28e9c5f3084c41eba9cb6460a02d8.pdf? ga $=2.8187383 .412999360 .1563384387-1677798579.1562816971$ 
Jain, P. (2006). Empowering Africa's development using ICT in a knowledge management approach. The Electronic Library, 24(1), 51-67.https://doi.org/10.1108/02640470610649245

Khalala, G., Makitla, I., Botha, A. y Alberts, R. (2014). A case for understanding user experience challenges confronting indigenous knowledge recorders in rural communities in South Africa. En IST-Africa Conference Proceedings (pp. 1-8). IEEE. https://doi.org/10.1109/ ISTAFRICA.2014.6880652

Loewen, J. y Dr. Kinshuk. (2012). The need for technological innovations for indigenous knowledge transfer in culturally Inclusive Education. Proceedings 4th International Conference on Advanced Learning Technologies, ICALT (pp. 577-578) IEEE. https://doi. org/10.1109/ICALT.2012.153

Maema, M., Terzoli, A. y Thinyane, M. (2013). A look into classification: Towards building an indigenous knowledge platform for educational use. En IST-Africa Conference \& Exhibition (pp. 1-8). IEEE.

McNulty, N. (2014). The Ulwazi programme: A case study in community-focused indigenous knowledge management. En K.-M. Osei-Bryson, G. Mansingh y L. Rao (Eds.), Knowledge management for development. Domains, strategies and technologies for developing countries (pp. 215-232). Springer. https://doi.org/10.1007/978-1-4899-7392-4

Mendoza, R. (2011). Generación y gestión del conocimiento permitido y del conocimiento necesario. Alianzas para el Empoderamiento Económico [red], 1-16. http://www.rimisp.org/ wp-content/files mf/1365018142articulo2generacionconocimiento.pdf

Monasterios, G. (2001). Aproximaciones al movimiento indígena en Internet. En 23 Congreso Internacional de la Latin American Studies Association (LASA) (pp. 6-8). www.mapuche.info/ mapuint/monasterios010906.pdf

Pérez, M.y Argueta, A. (2011). Saberes indígenas y diálogo intercultural.Cultura yepresentaciones Sociales, 5(10), 31-56. http://www.scielo.org.mx/scielo.php?script=sci arttext\&pid =S2007-81102011000100002

Reitmaier, T., Bidwell, N. J. y Marsden, G. (2011). Situating digital storytelling within African communities. International Journal of Human Computer Studies, 69(10), 658-668. https:// doi.org/10.1016/j.ijhcs.2010.12.008

Reitsma, L., Smith, A. y van den Hoven, E. (2013). StoryBeads: Preserving indigenous knowledge through tangible interaction design. En International Conference on Culture and Computing (pp. 79-85). IEEE. https://doi.org/10.1109/CultureComputing.2013.22 
http://doi.org/10.15359/ree.24-2.6

ROR: https://ror.org/01t466c14 Universidad Nacional, Costa Rica

http://www.una.ac.cr/educare

educare@una.cr

Rodil, K., Winschiers-Theophilus, H., Bidwell, N. J., Eskildsen, S., Rehm, M. y Kapuire, G. (2011). A new visualization approach to re-contextualize indigenous knowledge in rural Africa. En P. Campos, N. Graham, J. Jorge, N. Nunes, P. Palanque y M. Winckler (Eds.), Human-computer interaction - INTERACT, Part II, Lecture notes in computer sciencie, (Vol. 6947, pp. 297-314). Springer. https://doi.org/10.1007/978-3-642-23771-3 23

Sandoval-Forero, E. A. (2013). Los indígenas en el ciberespacio. Agricultura, Sociedad y Desarrollo, 10, 235-256. http://132.248.9.34/hevila/Agriculturasociedadydesarrollo/2013/vol10/ no2/6.pdf

Sierra, L. M., Naranjo, R. C. y Rojas, T. (2010). Una comunidad virtual de apoyo a procesos de etnoeducación para la comunidad nasa del resguardo de Corinto Lópezadentro, departamento del Cauca - Colombia. Revista Científica Guillermo de Ockham, 8(2), 101-114. https://doi.org/10.21500/22563202.567

Siew, S.-T., Yeo, A. W. y Zaman, T. (2013). Participatory action research in software development: Indigenous knowledge management systems case study. En M. Kurosu (Ed.), Humancomputer interaction. Human-centred design approaches, methods, tools, and environments. HCl. Lecture Notes in Computer Science (Vol. 8004, pp. 470-479). Springer. https://doi. org/10.1007/978-3-642-39232-0 51

Slavsky, L. (2007). TIC para jóvenes indígenas: Una experiencia mapuche en la creación colectiva de contenidos culturales digitales. Quinto Sol, 11, 143-166. https://doi.org/10.19137/ qs.v11i0.723

Sompong, N. y Rampai, N. (2015). The development model of knowledge management to strengthen Thai ict community learning center. Procedia - Social and Behavioral Sciences, 176, 139-147. https://doi.org/10.1016/j.sbspro.2015.01.454

Thapa, N. y Devi, J. S. (2015). Preservation of the traditional knowledge of tribal population in India. En 4th International Symposium on Emerging Trends and Technologies in Libraries and Information Services (pp. 99-103). IEEE. https://doi.org/10.1109/ETTLIS.2015.7048180

Thinyane, M., Dalvit, L., Slay, H., Mapi, T., Terzoli, A. y Clayton, P. (2007). An ontology-based, multimodal platform for the inclusion of marginalized rural communities into the knowledge society. En Annual Research conference of the South African Institute of Computer Scientists and Information Technologists on IT research in developing countries (pp. 143-151). ACM. https://doi.org/10.1145/1292491.1292508

Valbuena, T. (2009). Digital inclusion of indigenous people in Colombia, by the digitalization and safeguarding of their intangible cultural heritage. En Proceedings of the 3rd international conference on Theory and practice of electronic governance (pp. 407-408). ACM. 10.1145/1693042.1693133 
Winschiers-Theophilus, H., Bidwell, N. J., Chivuno-Kuria, S. y Kapuire, G. K. (2010). Determining requirements within an indigenous knowledge system of African rural communities. En Proceedings of the 2010 Annual Research Conference of the South African Institute of Computer Scientists and Information Technologists (pp. 332-340). ACM. https://doi. org/10.1145/1899503.1899540

Zaman, T., Winschiers-Theophilus, H., Yeo, A. W., Ting, L. C. y Jengan, G. (2015). Reviving an indigenous rainforest sign language: Digital Oroo'adventure game. En ICTD'15: Proceedings of the Seventh International Conference on Information and Communication Technologies and Development (Art. 69, pp. 1-4). ACM. https://doi.org/10.1145/2737856.2737885

Zaman, T., Yeo, A. W. y Kulathuramaiyer, N. (2013). Augmenting indigenous knowledge management with information and communication technology. International Journal of Services, Technology and Management, 19(1-2-3), 137-148. https://doi.org/10.1504/ IJSTM.2013.054202 